An Iterative Learning Control Approach for Radio Frequency Pulse Compressor Amplitude and Phase Modulation

Journal Article

Author(s):

Rezaeizadeh, Amin; Kalt, Roger; Schilcher, Thomas; Smith, Roy (D)

Publication date:

2016-04

Permanent link:

https://doi.org/10.3929/ethz-b-000111244

Rights / license:

In Copyright - Non-Commercial Use Permitted

Originally published in:

IEEE Transactions on Nuclear Science 63(2), https://doi.org/10.1109/TNS.2015.2463103 


\title{
An Iterative Learning Control Approach for Radio Frequency Pulse Compressor Amplitude and Phase Modulation
}

\author{
Amin Rezaeizadeh, Roger Kalt, Thomas Schilcher and Roy S. Smith
}

\begin{abstract}
Radio Frequency (RF) pulse compressors are used in linear accelerators (Linac) to achieve high power levels by shortening the RF pulse length. In their original form, the phase of the incoming pulse is reversed by $180^{\circ}$ which generates a high peak power at the output of the pulse compressor, followed by an exponential decay. This pulse shape however is not appropriate with regard to timing stability as well as for having equal energy gain for multi-bunch operation. To achieve flat-topped pulses, a method has been previously proposed in [1] which analytically modulates the input phase waveform. In the present contribution an alternative way to producing flat-topped RF pulses is proposed which is based on Iterative Learning Control technique. This approach manipulates the input waveforms iteratively in order to generate flat-topped amplitude and phase pulses at the output of the pulse compressor.
\end{abstract}

Index Terms-Linear accelerator, free electron laser, radio frequency control, pulse compressor, iterative learning control.

\section{INTRODUCTION}

$\mathbf{T}$ HE SwissFEL machine, currently being developed and constructed at Paul Scherrer Institut, will provide a source of bright and short X-ray pulses [2]. The SwissFEL C-band $(5.712 \mathrm{GHz})$ Linac includes 26 Radio Frequency (RF) stations. In each RF station, the klystron delivers high power RF to an RF pulse compressor followed by four accelerating structures [3]. The RF pulse compressor is a passive device used to store the energy and release it under certain conditions[1]. It essentially converts a long RF pulse to a short one with much higher peak RF magnitude. The SwissFEL RF pulse compressor is designed based on a single Barrel Open Cavity (BOC) which has a high quality factor resulting in a relatively long filling time and significant energy storage capacity (see Figure 1)[4]. In the original form of RF pulse compression, commonly referred to as the "phase jump" regime, the input phase is flipped by $180^{\circ}$, generating a reflected wave into the acceleration structures. This transient high power RF decays relatively slowly which gives enough time to the structures to build up an accelerating gradient much higher than using the klystron alone. However, this pulse shape is not suitable for multi-bunch operation where it is often required that all

Amin Rezaeizadeh is with Department of Electrical Engineering and Information Technology, ETH Zurich, CH-8092 Zurich, Switzerland, and also with the LLRF team at the Paul Scherrer Institut, AG-5232 PSI-Villigen, Switzerland (email: aminre@ee.ethz.ch).

Roger Kalt and Thomas Schilcher are with the Paul Scherrer Institut, AG5232 PSI-Villigen, Switzerland.

Roy S. Smith is with Automatic Control Laboratory (IfA), ETH Zurich, CH-8092 Zurich, Switzerland.

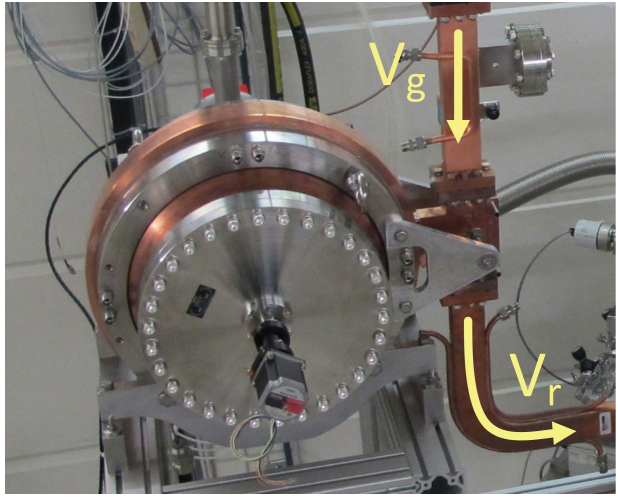

Fig. 1. The pulse compressor based on a single Barrel Open Cavity. The $V_{g}$ and $V_{r}$ denote the input and output forward voltages.

bunches experience the same RF amplitude and phase through the structures. Moreover, due to the spiky shape of the pulse, the resulting accelerating gradient is very sensitive to the timing jitters. To cope with this problem, a more complicated form of pulse compression was introduced which is referred to as the "phase modulation"[1]. In this method, the input phase is reversed very slowly so that the peak amplitude of the pulse compressor output is lowered and flattened.

The SwissFEL machine operate in a pulsed mode at the rate of $100 \mathrm{~Hz}$, using normal conducting structures. The RF pulse length is of the order of $1-3 \mu \mathrm{s}$ and no digital RF feedback loop is used within a pulse. Iterative learning control (ILC) is a control technique for systems that operate in a repetitive, or run-to-run, manner [6], [7], [8], [9], [10]. In this method, the measured trajectory is compared to the desired one to give an error estimate. The error is then used to update the inputs for the next run. For our problem, i.e. controlling the pulse waveform, an iterative control is a good approach since no intra-pulse feedback loop is feasible. Previously in [11], a model-free ILC algorithm was developed to control the klystron RF pulse [12]. In this paper, we develop and apply a model-based ILC approach for producing flat-topped RF pulse at the pulse compressor output. In this approach, both input phase and amplitude waveforms are modulated. The method has been successfully tested on the RF pulse compressor at the SwissFEL Linac test facility.

This paper provides more materials and additional analysis, not previously reported in [13]. The paper is organized as follows. Section II introduces the pulse compressor model 


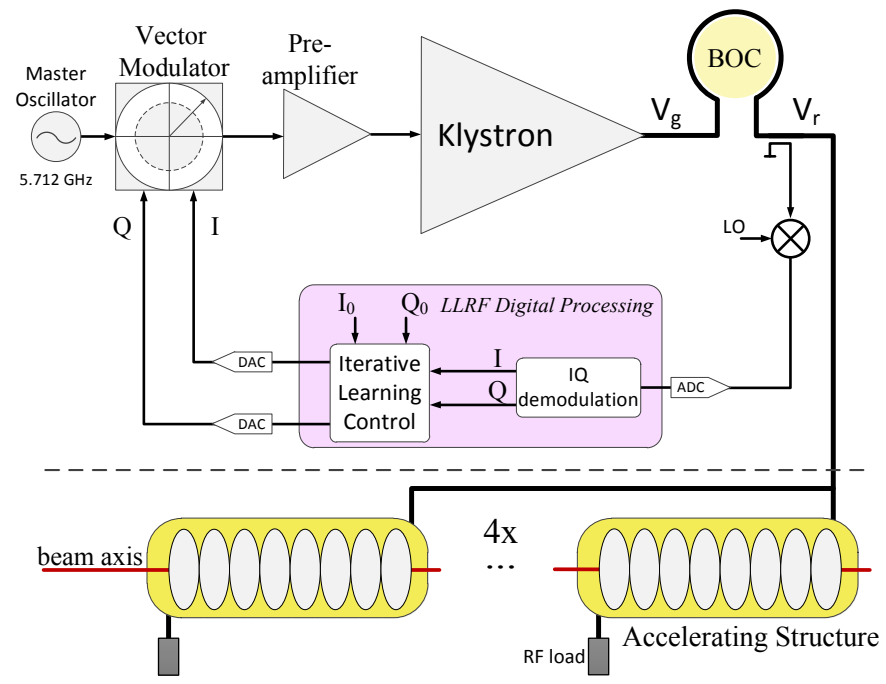

Fig. 2. The RF layout of a C-band station in the SwissFEL beamline.

and dynamics including an overview on the low-level RF architecture of a C-band RF station. The two conventional operation modes of pulse compressors are discussed in detail in Section III. Section IV develops the iterative learning control approach for amplitude and phase modulation, followed by the experimental results and a comparison with the phase modulation approach. Section VI concludes the paper with a summary of the results.

\section{SYSTEM DESCRIPTION}

The simplified layout of a C-band RF station in the SwissFEL Linac is illustrated in Figure 2. The C-band signal source is generated by a master oscillator. The discrete waveforms of the in-phase, $I$, and quadrature, $Q$, components of the RF signal are fed into the vector modulator to be up-converted. Each sequence contains 2048 samples with sampling time of 4.2 ns. The RF signal drives the klystron to generate high power RF at its output. The output signal is then fed to the pulse compressor to deliver a high peak power by shortening the RF pulse length. The high power signal is then split over four accelerating structures [3]. The RF field at the output of the BOC is measured by a directional coupler and downconverted to the baseband. The resulting signal is then sampled at the rate of $238 \mathrm{MHz}$, followed by a non-IQ demodulation algorithm to obtain the discrete sequences of $I$ and $Q$. The details of the low level RF architecture design are reported in [14]. The measured $I$ and $Q$ waveforms are used in the ILC algorithm to update the next $I$ and $Q$ input waveforms to the Digital-to-Analog Converters (DAC). The control objective of the ILC is to generate flat-topped RF amplitude and phase pulses at the pulse compressor output.

\section{A. The pulse compressor model}

The relationship between the klystron and pulse compressor voltage is given by [1]

$$
\alpha V_{g}=V_{c}+\tau \dot{V}_{c}
$$

where $V_{c}$ and $V_{g}$ are respectively the pulse compressor and klystron voltage phasors which are complex numbers containing the voltage magnitude and phase. Furthermore, $\alpha=\frac{2 \beta}{\beta+1}$, and $\beta$ is the coupling factor and $\tau$ is the filling time of the pulse compressor given by

$$
\tau=\frac{2 Q_{0}}{(\beta+1) \omega_{0}},
$$

where $Q_{0}$ is the unloaded quality factor and $\omega_{0}$ is the angular frequency of the RF wave. To derive Equation 1, it has been assumed that the unloaded quality factor is high enough, thus $1 / \omega_{0}$ can be neglected with respect to $\tau$. Furthermore, $V_{g}$ is assumed to be constant or to change smoothly (i.e. $\dot{V}_{g} \ll \omega_{0}$ ). For the case where the RF wave frequency and the pulse compressor resonant frequency are different, Equation 1 is replaced by[5]:

$$
\alpha V_{g}=V_{c}(1+j \tau \Delta \omega)+\tau \dot{V}_{c}
$$

where $\Delta \omega=\omega_{0}-\omega_{c}$, and where $\omega_{c}$ is the nominal angular resonant frequency of the pulse compressor. This frequency shift is introduced to remove the residual phase modulation by operating the klystron with a lower frequency than of the accelerating structure, as described in [1]. We refer to this operation as "detuning" the BOC. The reflected wave from the pulse compressor, which is the signal fed to the accelerating structure, is specified as the output voltage of the pulse compressor. The reflected voltage, given by $V_{r}=V_{c}-V_{g}$, is the quantity that we are interested in (see Figure 1).

\section{TWO MODES OF OPERATION}

In this section, we briefly survey the two common operation modes of pulse compressors, namely: the phase jump and the phase modulation.

\section{A. Phase Jump}

In this mode, the input RF phase is flipped by 180 degrees while the input amplitude is kept constant. This makes an amplitude spike at the output of the pulse compressor which decays slowly. However, this amplitude pulse shape is not suitable for multi-bunch operation of a Linac, since the bunches experience different accelerating gradients as they pass through the structure. As it has been proposed previously in [1], the output amplitude can be lowered and flattened by reversing the phase slowly. In this method, commonly known as the "phase modulation", the phase trajectory is derived analytically.

\section{B. Phase Modulation}

From Equation 1 and the fact that the reflected voltage is $V_{r}=V_{c}-V_{g}$, we lead to the following differential equation governing the system dynamics:

$$
V_{r}+\tau \dot{V}_{r}=(\alpha-1) V_{u}-\tau \dot{V}_{u} .
$$

For simplicity, the low-pass dynamics of the klystron and other LLRF devices are ignored. Therefore, $V_{u}$ denotes the voltage phasor introduced at the vector modulator. 
In this approach, the input amplitude is kept constant, i.e. we take $\left|V_{u}\right|=1$. Furthermore, the input phase also remains constant before starting the phase modulation. Hence, the initial input voltage is,

$$
V_{u}=1 e^{j 0}=1,
$$

with zero phase and unit amplitude.

The output voltage of the pulse compressor can be readily calculated using Equation 4,

$$
V_{r}+\tau \dot{V}_{r}=\alpha-1 .
$$

Thus, the reflected voltage rises exponentially,

$$
V_{r}(t)=\alpha-1-\alpha e^{-t / \tau},
$$

until it reaches,

$$
V_{r}\left(t_{0}^{-}\right)=\alpha-1-\alpha e^{-t_{0} / \tau},
$$

where $t_{0}$ is the beginning time of the phase modulation, or in the phase jump regime, it is the time when the phase is flipped by $180^{\circ}$.

At the time $t=t_{0}$, the input phase jumps to $\phi_{u}\left(t_{0}\right)$ which is less than 180 degrees,

$$
V_{u}\left(t_{0}^{+}\right)=1 e^{j \phi_{u}\left(t_{0}\right)}
$$

From the equation dynamics in (4), we have a direct feedthrough term of -1 , i.e. the change of $V_{u}$ is opposite to the change of $V_{r}$. This implies that any changes in the input voltage are directly translated to the output by the feed-through term. Therefore, at the time $t_{0}$ when the phase modulation starts, there is a jump in the output voltage determined as follows,

$$
\begin{aligned}
V_{r}\left(t_{0}^{+}\right)= & V_{r}\left(t_{0}^{-}\right)+\Delta V_{r}\left(t_{0}\right)=V_{r}\left(t_{0}^{-}\right)-\Delta V_{u}\left(t_{0}\right) \\
& =V_{r}\left(t_{0}^{-}\right)+\left(1-e^{j \phi_{u}\left(t_{0}\right)}\right)=V_{0} e^{j \phi_{r}\left(t_{0}\right)},
\end{aligned}
$$

where $V_{0} \in \mathbb{R}$ and $\phi_{r}\left(t_{0}\right)$, denote respectively the amplitude and phase of the output voltage at time $t_{0}$. Since the output amplitude is to be constant, it can be expressed as

$$
V_{r}(t)=V_{0} e^{j \phi_{r}(t)},
$$

for $t>t_{0}$ until the end of the klystron pulse. Substituting Equation 11, the input voltage and the derivatives into the differential equation (4), yields

$V_{0} e^{j \phi_{r}(t)}+\tau V_{0} j \dot{\phi}_{r}(t) e^{j \phi_{r}(t)}=(\alpha-1) e^{j \phi_{u}(t)}-\tau j \dot{\phi}_{u}(t) e^{j \phi_{u}(t)}$.

Separating the imaginary and real terms, we have,

$$
\begin{aligned}
V_{0} & =(\alpha-1) \cos \left(\phi_{u}-\phi_{r}\right)+\tau \dot{\phi}_{u} \sin \left(\phi_{u}-\phi_{r}\right) \\
\tau V_{0} \dot{\phi}_{r} & =-\tau \dot{\phi}_{u} \cos \left(\phi_{u}-\phi_{r}\right)+(\alpha-1) \sin \left(\phi_{u}-\phi_{r}\right),
\end{aligned}
$$

where the time index is removed for notational simplicity. Equations 13 are nonlinear first order differential equations which can be solved numerically with the initial conditions on $\phi_{u}\left(t_{0}\right)$ and $\phi_{r}\left(t_{0}\right)$. The value for $V_{0}$ is already calculated in Equation 10.

Figure 3 illustrates the applied input signals used in phase jump and phase modulation modes. In both methods, the input
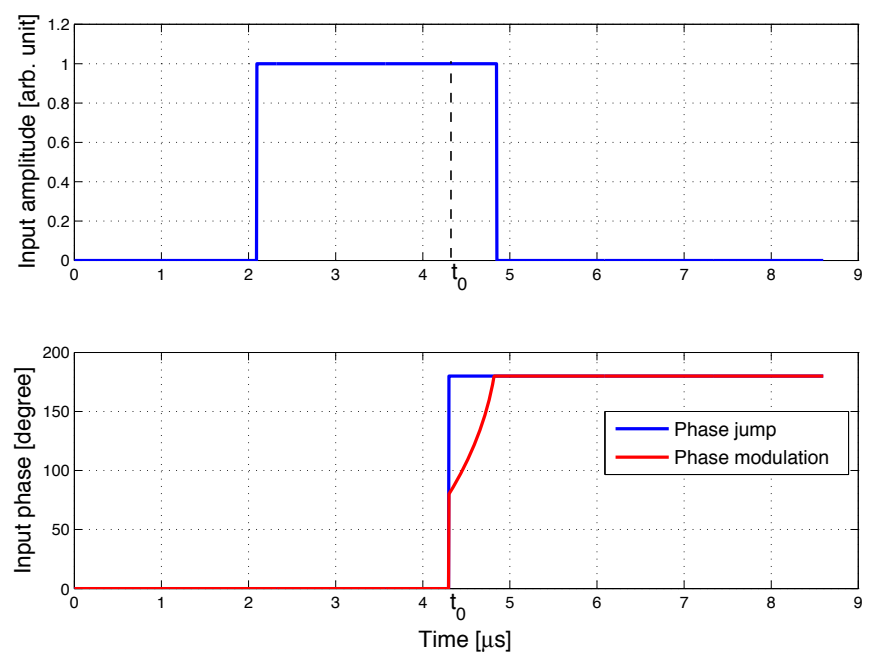

Fig. 3. The input amplitude and phase waveforms for the two modes of phase jump and phase modulation regime.
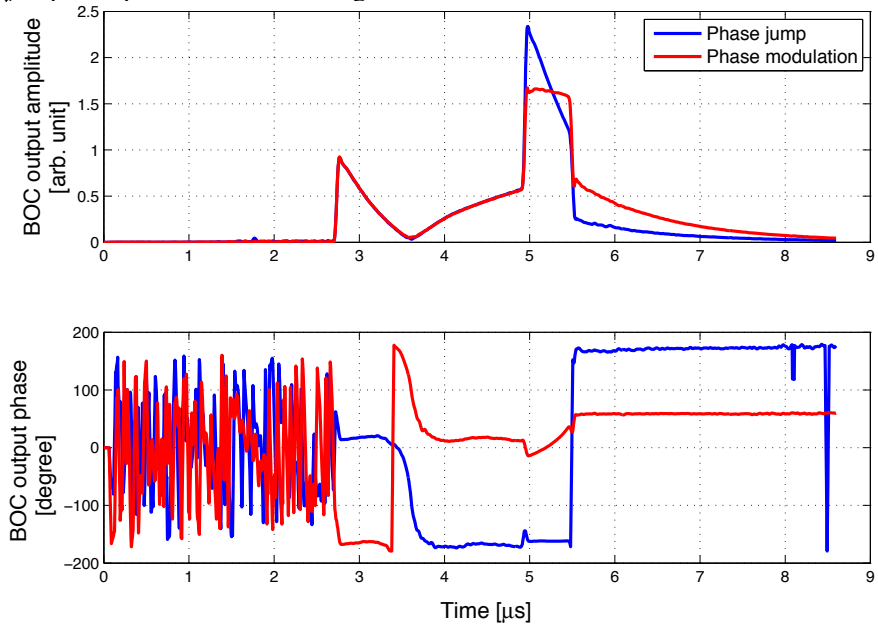

Fig. 4. The pulse compressor output amplitude and phase waveforms for the two modes of phase jump and phase modulation regime.

amplitude remains constant. The input phase trajectory in phase modulation mode, shown in Figure 3, is the solution to the differential equation in (13). The measured output signals are plotted in Figure 4. As we can see, the BOC output amplitude is lowered and relatively flattened. However, since the dynamics of the klystron and the RF drive chain are usually ignored in the analytical approach, the output amplitude is not perfectly flat. As stated earlier, the output phase in the phase modulation regime, can be flattened by operating the klystron with a lower frequency than of the accelerating structure. This can be achieved by adding a linear phase to the input phase waveform of the klystron. The resonant frequency of the pulse compressor should be slightly moved according to the introduced frequency shift. The resonant frequency of the BOC is controlled via the body temperature.

\section{ITERATIVE LEARNING SCHEME}

In the phase modulation regime, the model-system mismatch leads to non-flat pulses. In this section, we develop an iterative learning control algorithm to improve the RF pulse 
flatness. In the proposed ILC approach, the algorithm starts off with the phase jump mode and iteratively updates the input waveforms to achieve flat-topped amplitude and phase pulses.

Discretizing equation (3) with Euler backward method (with sampling time $T_{s} \ll \tau$ ) and taking the Z-transform, lead us to the transfer function, relating the klystron voltage to the output voltage of the BOC:

$$
G_{B O C}(z)=\frac{V_{r}(z)}{V_{g}(z)}=\frac{T_{s}(\alpha-1)-\tau-j T_{s} \tau \Delta \omega+\tau z^{-1}}{T_{s}+\tau+j T_{s} \tau \Delta \omega-\tau z^{-1}} .
$$

We model the RF drive chain, including the vector modulator and pre-amplifier, as a 1st-order low pass system with a bandwidth determined by $\gamma$ and a scalar gain $K$ that can be complex in general to capture the loop phase. Therefore, the overall transfer function from the DAC inputs to the measured voltage at the BOC output is modeled as

$$
G(z)=K \frac{1-\gamma}{1-\gamma z^{-1}} G_{B O C}(z) .
$$

Using the lifted system representation, the system equations can be formulated as

$$
y_{I}+j y_{Q}=G_{I Q}\left(u_{I}+j u_{Q}\right),
$$

where $u_{I}, u_{Q}, y_{I}$ and $y_{Q} \in \mathbb{R}^{N}$ are, respectively, the DAC $I$ and $Q$ waveforms and the measured $I$ and $Q$ waveforms at the BOC output. The matrix $G_{I Q} \in \mathbb{R}^{N \times N}$ is the lowertriangular Toeplitz matrix of the impulse response $h(k)$ derived from Equation 15, i.e.,

$$
G_{I Q}=\left(\begin{array}{cccc}
h(1) & 0 & \cdots & 0 \\
h(2) & h(1) & \cdots & 0 \\
\vdots & \vdots & \ddots & \vdots \\
h(N) & h(N-1) & \cdots & h(1)
\end{array}\right),
$$

where $N$ denotes the number of samples in the flat-topped region. The $G_{I Q}$ can be split into real and imaginary parts as $G_{I Q}=G_{r}+j G_{i}$, where $G_{r}$ and $G_{i}$ are real matrices. Hence, the system equations are given by

$$
\begin{aligned}
y_{I} & =G_{r} u_{I}-G_{i} u_{Q} \\
y_{Q} & =G_{i} u_{I}+G_{r} u_{Q} .
\end{aligned}
$$

Stacking the $I$ and $Q$ terms into super-vectors, we get the following expression for the system input-output relationship,

$$
y=G u+d,
$$

where,

$$
y:=\left(\begin{array}{c}
y_{I} \\
y_{Q}
\end{array}\right), \quad u:=\left(\begin{array}{c}
u_{I} \\
u_{Q}
\end{array}\right), \quad G:=\left(\begin{array}{cc}
G_{r} & -G_{i} \\
G_{i} & G_{r}
\end{array}\right) .
$$

and where $d$ is the output disturbance which we assume it also captures the uncertainty about the system. We denote by subscript $i$ to be the iteration counter to identify signals from different iterations. At trial $i^{t h}$, the measured output is given by

$$
y_{i}=G u_{i}+d,
$$

Where we assume that the output disturbance term is trialinvariant. The algorithm proposed here calculates the control input for the $(i+1)$-th iteration as the solution of an optimization problem,

$$
\begin{aligned}
u_{i+1}=\operatorname{argmin} & \left\{J_{i+1}\left(u_{i+1}\right):\right. \\
& \left.e_{i+1}=y_{d}-y_{i+1}, y_{i+1}=G u_{i+1}+d\right\},
\end{aligned}
$$

and the pulse flatness objective at iteration $i+1$, is expressed in terms of the following cost function,

$$
J_{i+1}\left(u_{i+1}\right)=\left\|y_{d}-y_{i+1}\right\|_{Q}^{2}+\left\|u_{i+1}-u_{i}\right\|_{R}^{2},
$$

where $\|\cdot\|$ are the weighted norms, and where $y_{d}$ denotes the desired output vector which is given by the desired $I$ and $Q$ waveforms:

$$
y_{d}=\left(\begin{array}{c}
y_{d I} \\
y_{d Q}
\end{array}\right)=\left(\begin{array}{c}
a_{d} \cos \varphi_{d} \\
a_{d} \sin \varphi_{d}
\end{array}\right),
$$

where $a_{d}$ and $\varphi_{d}$ are respectively the desired output amplitude and phase in the flat-topped region. We choose a constant $\varphi_{d}$ over the flat-topped region, while the desired amplitude is smoothed and thus time-dependent (see [11]),

$a_{d}(k)=a_{0} e^{-(k-1) / k_{0}}+a_{r e f}\left(1-e^{-(k-1) / k_{0}}\right), \quad 1 \leq k \leq N$,

where $a_{r e f}$ is the desired amplitude at the flat-topped region, and $a_{0}$ and $k_{0}$ are some constants.

Furthermore, the disturbance term can be estimated from the previous trial:

$$
d \simeq y_{i}-G u_{i} .
$$

Substituting Equation 25 into the cost function and removing the terms independent of $u_{i+1}$, leads to

$$
\begin{aligned}
\tilde{J}_{i+1}\left(u_{i+1}\right)= & u_{i+1}^{T}\left(R+G^{T} Q G\right) u_{i+1}+ \\
& -2\left(u_{i}^{T} R+\left(y_{d}-y_{i}+G u_{i}\right)^{T} Q G\right) u_{i+1},
\end{aligned}
$$

which gives the optimal control input for the next iteration:

$$
u_{i+1}=u_{i}+\left(R+G^{T} Q G\right)^{-1} G^{T} Q\left(y_{d}-y_{i}\right), \forall i \geq 0 .
$$

Note that the weight matrices $R$ and $Q$ can be trial-variant, but in order to reduce the computational burden we take them constant. Thus, the inverse of the matrix is calculated once and stored. Of course there should be upper and lower bounds on the input signals (for both $I$ and $Q$ input waveforms). That is,

$$
u_{\text {low }} \leq u_{i+1} \leq u_{\text {up }} .
$$

Throughout this section, we assumed that the input-output delay is already taken into account. The delay of the channel must be precisely measured, otherwise the algorithm may run into instability. The iterative control approach is summarized in Algorithm 1.

\section{EXPERIMENTAL RESULTS}

The experiment parameters are given in Table (I). For this experiment, the pulse compressor is detuned, as per (3). Figure 5 illustrates the model response and the measured BOC output $I$ and $Q$ signals. The flat-topped region, where the ILC applies, is colored and it contains $N$ samples. The iterative 


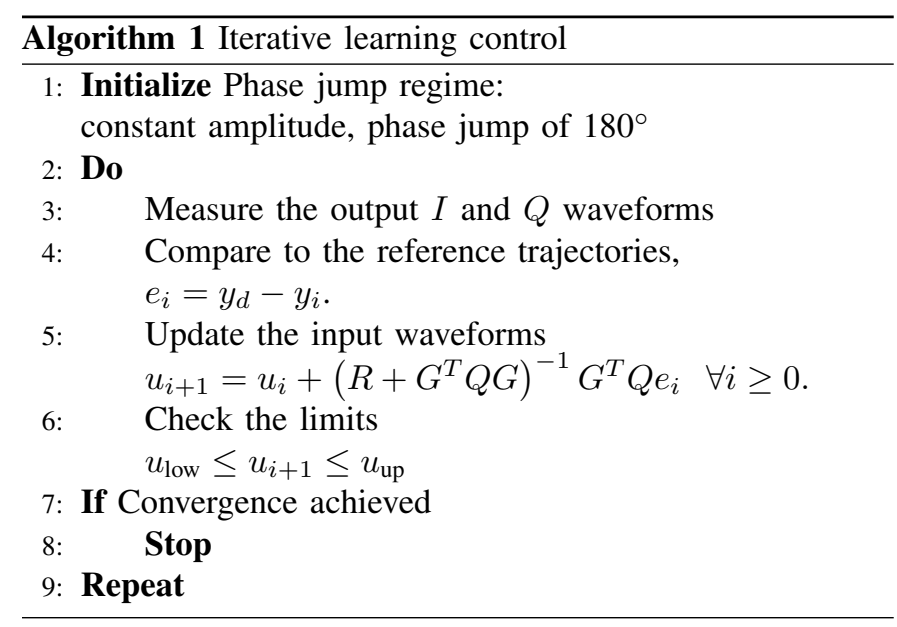

TABLE I

EXPERIMENT PARAMETERS

\begin{tabular}{lc}
\hline \hline Parameter & value \\
\hline$\beta$ & 10.33 \\
$Q_{0}$ & 220085 \\
$\omega_{0}$ & $2 \pi \times 5.712 \mathrm{GHz}$ \\
$\tau$ & $1.086 \mu \mathrm{s}$ \\
$\gamma$ & 0.7 \\
$\Delta \omega$ & $250 \mathrm{kHz}$ \\
$T_{s}$ & $4.2 \mathrm{~ns}$ \\
$N$ & 120 \\
\hline \hline
\end{tabular}

learning algorithm is initialized with the phase jump mode. The initial input to the DAC table is the phase jump waveforms with an additional linear phase with the slope proportional to the detuning frequency, $\Delta \omega$. The input phase waveform is iteratively modified from a $180^{\circ}$ sharp step to a smoothly reversed phase. Here, the klystron is operated slightly below the saturation to give enough headroom for amplitude modulation. Between each iteration, 10 output waveforms are captured and filtered to suppress both random noise as well as the repetitive non-IQ demodulation patterns. Since at each iteration the amplitude pulse shape is modified, some amount of time should be considered for the BOC temperature stabilization unit. Especially, during the early iterations, the waiting time between two consecutive iterations is relatively large, as the amplitude waveform changes significantly. Figure 6 shows the experimental result of the ILC-based method (after 20 iterations) and the comparison with the phase modulation method. The difference in waveforms, generated by phase modulation, between Figure 6 and 4 comes from detuning the pulse compressor. The updated input amplitude and phase waveforms are plotted in Figure 7. The output phase can be changed by adding an offset to both input phase waveform and the desired flat-top phase $\varphi_{d}$. According to Figure 7, there is no significant change in input average power. Figure 10 illustrates the standard deviation of amplitude and phase pulses over the flat-topped region, as a good measure of pulse flatness. Comparing the performance of the two methods of ILC and phase modulation, the relative standard deviation of amplitude is reduced by a factor of 9 and for the phase by
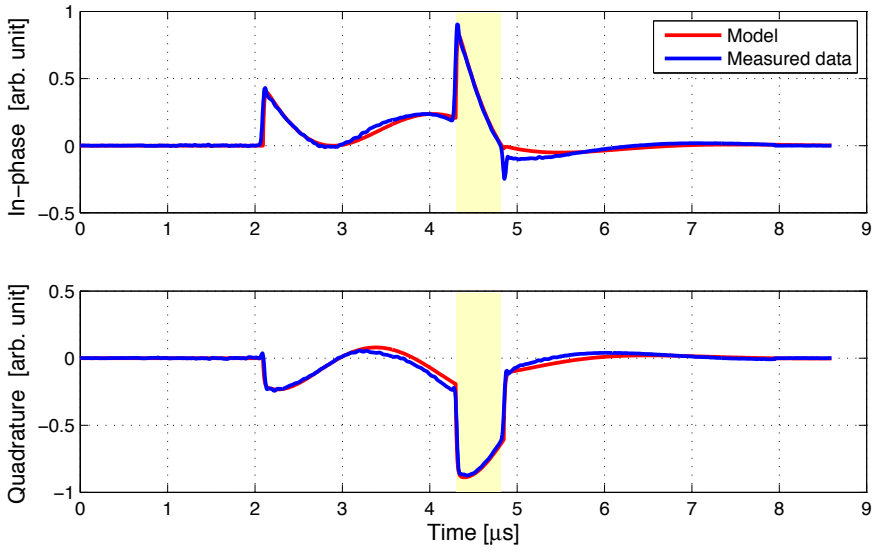

Fig. 5. The model and the BOC output measured $I$ and $Q$ signals. The region where the model applies is colored. The number of samples is 2048 with the sampling time of $T_{s}=4.2 \mathrm{~ns}$.
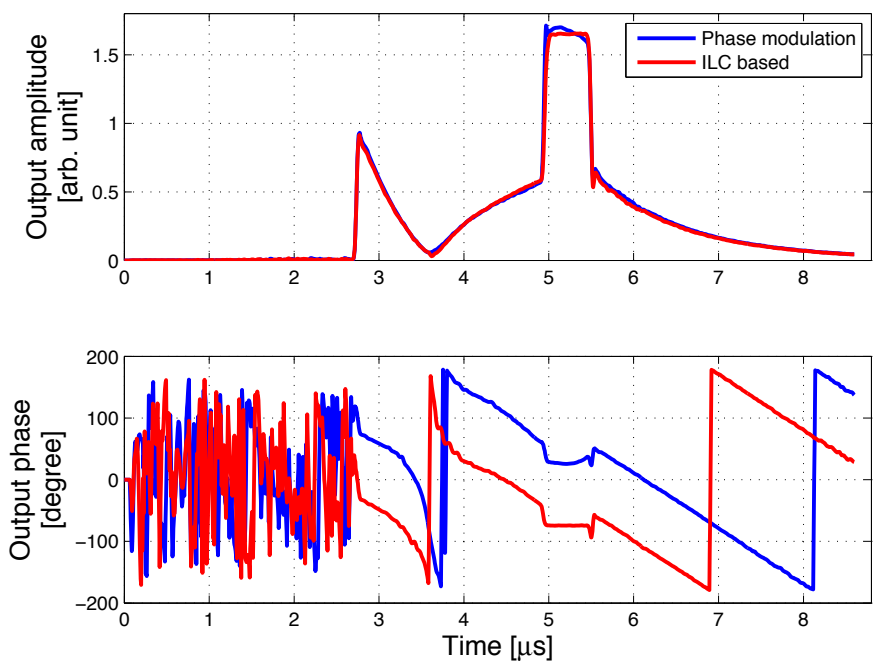

Fig. 6. The BOC output amplitude and phase waveforms of the ILC-based and the phase modulation methods on the detuned pulse compressor.

a factor of 12 in the ILC-based approach. Figures 9 and 10 , respectively, illustrate the RF amplitude and phase over the flat-topped region for different number of iteration, and a comparison with the phase modulation approach. The phase offset is removed to compare the two methods. As the iteration proceeds, the waveforms approach the desired trajectory (as shown in red for the amplitude). The desired phase waveform was simply chosen to be constant over the flat-topped region, even though the algorithm works for any arbitrary waveform.

\section{CONCLUSION}

We proposed an iterative learning approach for pulse shaping in which the measured output $I$ and $Q$ waveforms are used to estimate the error of flatness. The algorithm iteratively updates the DAC input $I$ and $Q$ waveforms for the next pulse. With the choice of $I$ and $Q$, instead of amplitude and phase, we benefit from linearity of the system dynamics. In the proposed ILC-based pulse flattening, in contrast to the phase modulation regime which is an open-loop analytical approach, the klystron 

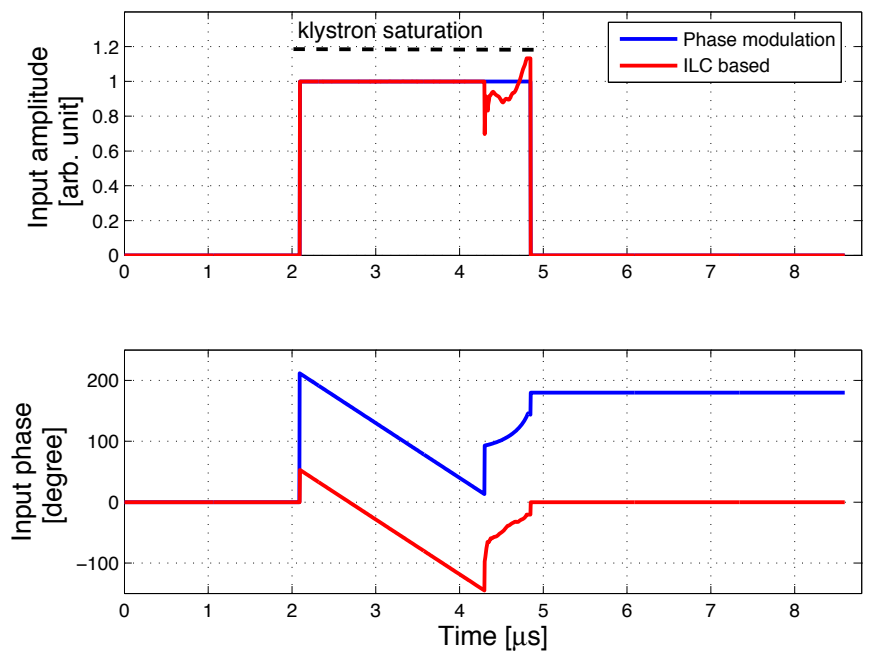

Fig. 7. The klystron input amplitude and phase waveforms for the two methods of phase modulation and ILC. The dashed line denotes the klystron saturating amplitude.
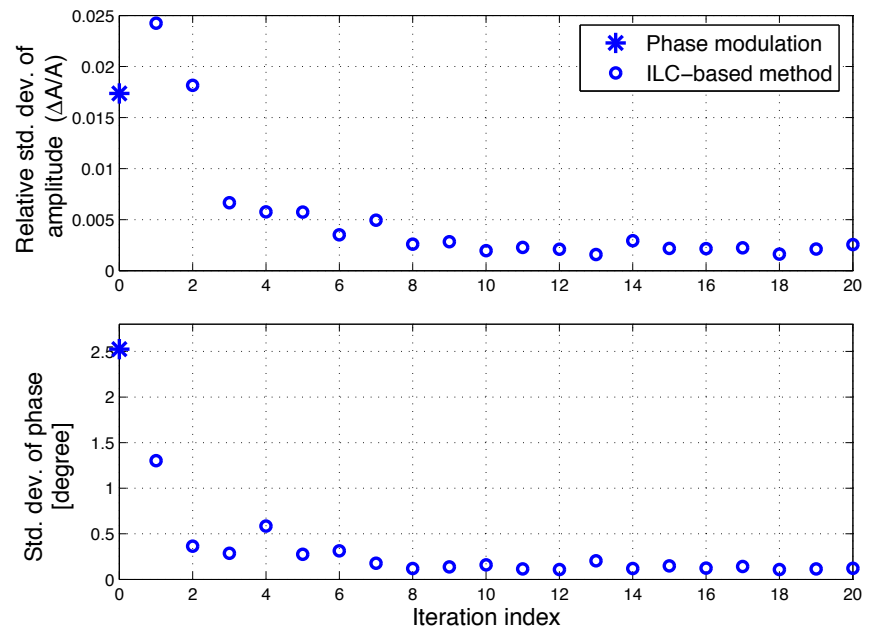

Fig. 8. The standard deviation of the BOC output amplitude and phase pulses over the flat-topped region.

amplitude is also modulated. The greatly improved pulse flattening comes at a cost. Simulation studies show that the energy gain drops by nearly $20 \%$ with the flat-topped RF pulse compared to the phase jump mode.

\section{ACKNOWLEDGMENT}

The authors would like to thank the LLRF and the RF team at Paul Scherrer Institut who made this research possible. Special thanks goes to Riccardo Zennaro and Alessandro Citterio for providing materials for the study.

\section{REFERENCES}

[1] A. Fiebig and Ch. Schieblich, "A SLED type pulse compressor with rectangular pulse shape", Proceedings of European Particle Conference, Nice, 1990 , p. 937.

[2] Romain Ganter et al., SwissFEL Conceptual Design Report, PSI, Villigen, Switzerland, 2010.

[3] R. Zennaro et al., "Conceptual design of the C-band module for the SwissFEL", Proceedings of Linear Accelerator Conference, Tsukuba, Japan, 2010.

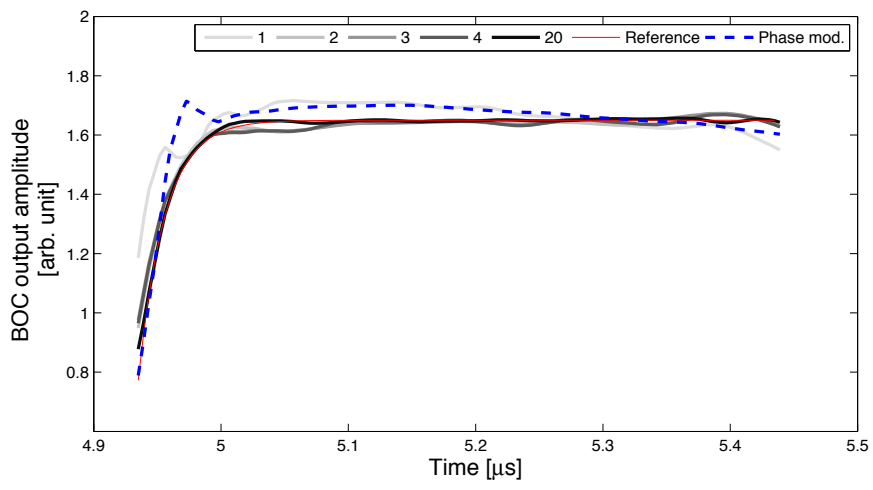

Fig. 9. The amplitude waveform at the flat-top region for different iterations. The desired amplitude waveform is depicted in red. The phase modulation method gives the dashed blue trajectory.

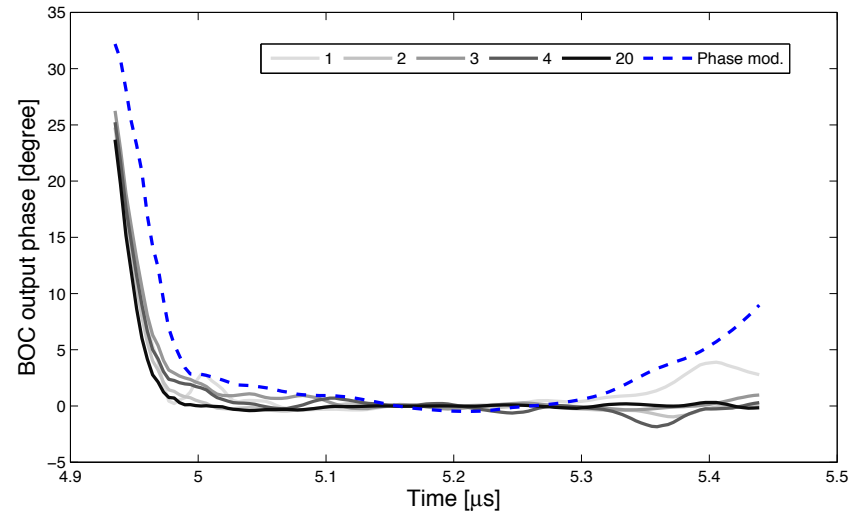

Fig. 10. The phase waveform at the flat-top region for different iterations. The offsets are removed in order to compare the two methods of ILC-based and the phase modulation approach.

[4] R. Zennaro, M. Bopp, A. Citterio, R. Reiser, T. Stapf, "C-band RF pulse compressor for SwissFEL", International Particle Accelerator Conference, Shanghai, China, 2013.

[5] S.H. Shaker et al., "Phase modulator programming to get flat pulses with desired length and power from the CTF3 pulse compressors", International Particle Accelerator Conference, Kyoto Japan, 2010.

[6] N. Amann et al., "Iterative learning control for discrete-time systems with exponential rate of convergence", IEE Proceedings Control Theory and Applications, 1996, 143, 217-224.

[7] Amann N. et al., "Iterative learning control using optimal feedback and feedforward actions", Proceedings of the 34th IEEE Conference on Decision and Control, New Orleans, LA, 1996.

[8] S. Arimoto, S. Kawamura, and F. Miyazaki, "Bettering operations of robots by learning", Journal of Robotic Systems, 1(2):123-140, 1984.

[9] D. A. Bristow, M. Tharayil, and A. G. Alleyne, "A survey of iterative learning control”, IEEE Control Systems Magazine, 26(3):96114, 2006.

[10] M. Uchiyama, "Formulation of high-speed motion pattern of a mechanical arm by trial", Trans. SICE (Society of Instrument and Control Engineers), 14(6):706-712, 1978 (in Japanese).

[11] A. Rezaeizadeh et al.,"RF pulse flattening in the SwissFEL test facility based on model-free iterative learning control", Proceedings of the 36th International Free Electron Laser Conference, Basel, Switzerland, August 2014.

[12] P. Janssens, G. Pipeleers, and J. Swevers, "Model-free iterative learning control for LTI systems with actuator constraints", Proceedngs of the 18th IFAC World Congress, pp. 11556-11561, Milano, Italy, 2011.

[13] A. Rezaeizadeh et al., "Pulse Compressor phase and amplitude modulation based on iterative learning control", Proceedings of the 6th International Particle Accelerator Conference, Richmond VA, USA, 2015.

[14] A. Hauff and et al. , "SwissFEL C-band LLRF Prototype System", 27th Linear Accelerator Conference, Geneva, Switzerland, 2014. 